\title{
The collapse of cooperation: the endogeneity of institutional break-up and its asymmetry with emergence
}

\author{
Christian Cordes $^{1}$ (1) - Wolfram Elsner ${ }^{1} \cdot$ Claudius Graebner $^{2,3}$. \\ Torsten Heinrich $^{4}$. Joshua Henkel ${ }^{1}$. Henning Schwardt ${ }^{5}$. Georg Schwesinger ${ }^{1}$. \\ Tong-Yaa Su ${ }^{1}$
}

Accepted: 19 July 2021 / Published online: 13 August 2021

(C) The Author(s) 2021

\begin{abstract}
Decline and break-up of institutionalized cooperation, at all levels, has occurred frequently. Some of its concomitants, such as international migration, have become topical in the globalized world. Aspects of the phenomenon have also become known as failing states. However, the focus in most social sciences has been on institutional emergence and persistence, not collapse. We develop an endogenous explanation of collapsing institutions. Collapse may be an implication of the very economic success of institutionalized cooperation and of increasing system complexity, when cognitive conditions for effective collective decisionmaking do not proportionately evolve. Moreover, we show that collapse is not a simple logical reverse of emergence. Rather, institutions break up at different factor constellations than the ones prevailing at emergence. We approach endogenous institutional break-up and its asymmetry from various paradigmatic and disciplinary perspectives, employing psychology, anthropology, network analysis, and institutional economics. These perspectives cover individuals, groups, interaction-arenas, populations, and social networks.
\end{abstract}

Keywords Anthropology · Cognitive capacities · Cooperation · Economic decline · Game theory $\cdot$ Institutional emergence $\cdot$ Network analysis $\cdot$ Social institutions

JEL Classifications A12 $\cdot \mathrm{B} 52 \cdot \mathrm{C} 72 \cdot \mathrm{D} 02 \cdot \mathrm{D} 03 \cdot \mathrm{L} 14 \cdot \mathrm{O} 15 \cdot \mathrm{O} 43 \cdot \mathrm{Z} 13$

\section{Introduction}

Collapse of institutional structures has been ubiquitous over thousands of years of human history (e.g., Turchin 2003; Diamond 2005; Tainter 2006; Acemoglu and Robinson 2012). Recently, the collapse of the state-socialist countries of Eurasia

Christian Cordes

c.cordes@uni-bremen.de

Extended author information available on the last page of the article 
in the 1990s and its socio-economic costs, a number of failing states, the apparent fragility of the entire global system, or the reduced collective-action capacity of the leading powers, when it comes to the maintenance of the global commons (e.g., Block 2000; Rasmus 2016), have become topical. Below entire systemic collapse, there usually are symptoms of institutional decline, at global, national or local levels. Examples include long-term economic downgrade, cumulative income inequality, social disintegration, or geographical fragmentation as well as symptoms of specific humanitarian crises as to food, health, water, land or shelter access, violence and wars, and subsequent enforced international migrations.

Yet, the focus of evolutionary and institutional economics has been on the emergence of cooperation. ${ }^{1}$ Considerations of collapse have been considerably fewer, and if so, only recent (e.g., Acemoglu and Robinson 2012; Horiuchi 2015). The issue was largely left to other social sciences, particularly history, ethnography, anthropology, social geography (Weiss and Bradley 2001; Tainter 2003, 2006; Brooks 2012; Meija 2018), or law and business (Richman 2016). However, these accounts consider the reasons for collapse as exogenous: through the failure of a political system, mismanagement by elites, or through climatic factors, particularly ecological disasters. Sometimes, as in Tainter (2003) and Diamond (2005), a population's failure to adapt to climate change takes effect, but the main explanation draws on exogenous factors. Few scholars have explicitly taken institutional aspects into account, such as biased collective decision-making in the presence of sunk costs of institutions (Janssen et al. 2003). In this paper, we will consider relatively low interaction costs of established institutions and relatively high interaction costs under institutional break-up and change, even if such change were appropriate and warranted. More generally, most historical instances, in which a complex society disintegrated and developed into less complex ones, where complexity and adaptability of collective decision-making fell below a degree required for the maintenance of the larger, changing commons, have been candidates for collapse (Tainter 2003; Turchin et al. 2013). Societies that are insufficiently complex or adaptive, when encountering change, face high interaction costs (Meija 2018). This causes a fragmentation of populations into smaller groups, constraining communication and interaction, and reducing higher-level collective-decision capacities, eventually leading to collapse.

This article contributes to that still small but growing literature on the degeneration, inadequacy, and eventual collapse of institutionalized cooperation, by adopting an interdisciplinary and multiparadigmatic perspective. It is motivated by two key theses: First, collapse is not necessarily tied to exogenous events, but might well occur because of endogenous dynamics. This resembles the idea of "improving oneself into extinction" (Elster 1983, 54), which was applied to an individualistic rationality in ubiquitous social dilemmas. It also applies to the development of populations, when the reach, complexity, or adaptability of collective decision-making is no longer appropriate to the size, complexity, and change of the socio-economic and natural conditions,

\footnotetext{
${ }^{1}$ Contributions here come from various fields: e.g. Axelrod (1984) for the evolution-of-cooperation approach, Hodgson (2000) for original institutionalist contributions, Epstein and Axtell (1996) for complexity sciences, or Boyd and Richerson (1985) for the anthropological approach.
} 
namely of their commons, and when this stems from the very success of the earlier institutionalized coordination of the population, but these very institutions have at some point become inadequate. Second, the collapse of cooperation is typically not merely a reversal of its emergence. Otherwise, its theoretical comprehension would be a simple derivative of the work on the latter. Rather, as the various disciplinary perspectives developed below indicate, collapse follows different rules than-and is asymmetric to-emergence. Hence, it should be a subject of investigation in its own right.

Such an investigation is necessarily interdisciplinary and considers different levels of analysis. Therefore, the following sections integrate arguments from various disciplines (or scientific paradigms) that study emergence and collapse in specific contexts. They focus either on the individual ('micro'), the group ('meso'), or systemic ('macro') level. To facilitate the triangulation of these contributions, an overall methodological framework is helpful. Given its generality and explicit consideration of the micromeso-macro distinction, we refer to the framework of 'systemism', which has originally been proposed by Mario Bunge (2004). It is widely used in both the social and natural sciences and has been used as an overall methodological framework for interdisciplinary and multiparadigmatic analysis (e.g. Gräbner and Kapeller 2017).

To investigate the claims on endogeneity and asymmetry of collapse, we proceed by discussing the arguments from the different perspectives one by one: the biology and evolutionary psychology of the individual (Sect. 3), the anthropology of group behavior (Sect. 4), network science (Sect. 5), and evolutionary institutionalism applying evolutionary game theory (Sect. 6). At the end of each section, we derive hypotheses about the mechanisms and dynamics of collapse, i.e., its endogeneity and/or asymmetry. In Sect. 7, we integrate the perspectives within a common systemist framework and show that they in fact converge to a theoretical frame for modeling, where collapse is endogenous and asymmetric to emergence.

\section{On the endogeneity and asymmetry of collapse}

This section elaborates on the two main claims introduced above, namely that, first, decline, crisis, and collapse of large-scale institutional cooperation will often turn out to be some endogenous implication of its very success and, second, that it will not be a simple logical reverse of emergence.

\subsection{The endogeneity of collapse}

It can be illustrated, based on some formal modeling (see Sect. 6), that institutional emergence-which is here understood as the emergence of a set of social rules that allow individuals to solve collective decision problems, including social dilemmas $^{2}$ - is unavoidably subject to interactive learning, trial and error in evolutionary processes as well as some risk-taking, as it implies some potential early disadvantage

\footnotetext{
${ }^{2}$ See the appendix for a table that contains an overview of the definitions of central concepts used in the paper.
} 
of the behavioral innovator compared to followers and imitators. Moreover, institutional emergence is subject to some broader and longer-run perspective, and, finally, to some generalization of experience into one's expectations and habituation. Institutional emergence also implies unavoidable sunk fixed costs of individual and collective decision-making (e.g., Elsner and Schwardt 2014). Therefore, some institutional hysteresis may easily become prevailing at some later point, since it is easier for agents to stick to the prevalent institution rather than interactively learn a new institution incurring new sunk fixed costs (e.g., Setterfield 1996).

Social institutions that are supposed to solve ubiquitous social dilemmas and collective action problems logically cannot be based on short-term maximization behavior. Institutions are to be applied semi-consciously, as long as there is no particular reason to expect that the next interaction partner will defect. Such learning and habituation imply a general relative persistence and stability of institutionalized behavior over more volatile socio-economic variables and some hysteresis, i.e., the institution's use beyond the point where it was still appropriate. Thus, some institutional degeneration may occur: for some shorter or longer time, the established institution still may be appropriately problem-solving. And even if it becomes increasingly normatized and detached from the original basic problem structure, it still may be an appropriate norm. But it will sooner or later become dysfunctional and eventually an improper abstract norm (e.g., Elsner 2012; Sugiarto et al. 2015). Sticking to the old, now petrifying institution then increasingly causes inappropriate decision-making and divert its carrier group from problem-solving, in this way entailing an institutional ceremonialization (Veblen 1899) of a formerly functional, instrumental institution. Other motives come to the fore rather than collective problem-solving, such as redistribution of income, power, or status among individuals or groups, or bureaucratic hierarchization. Such increasing dysfunctionality causes eventual collapse.

This may be a consequence of cooperation success, an endogenous process also in another respect. The instrumental institution promoted effective decision-making and socio-economic success. Economy and society, and their commons, may have grown in size and numbers, affording greater differentiation and heterogeneity of agents and their relations. A higher systemic complexity can then be accommodated for some time facilitated by the reduction of the complexity of the individual decision situation through the very institution. However, as more agents are integrated, problems can arise for both newcomers and incumbents. Institutions should adapt to accommodate larger numbers and more heterogeneity. Yet, cognitive conditions (changing experience and expectations) for both types of agents may deteriorate with increasing numbers, heterogeneity, and perceived increasing complexity and turbulence. Consequently, cognitive limits become binding. If proper institutional adaptation will not or only insufficiently happen, experience and expectation of trust and trustworthiness deteriorate and cooperation may collapse.

\subsection{The asymmetry of collapse}

As to asymmetry, there is a turning point from institutional emergence, stabilization, and instrumental functionality toward ceremonial inappropriateness, decreasing performance, and eventual collapse. When and how does non-linearity (asymmetry) 


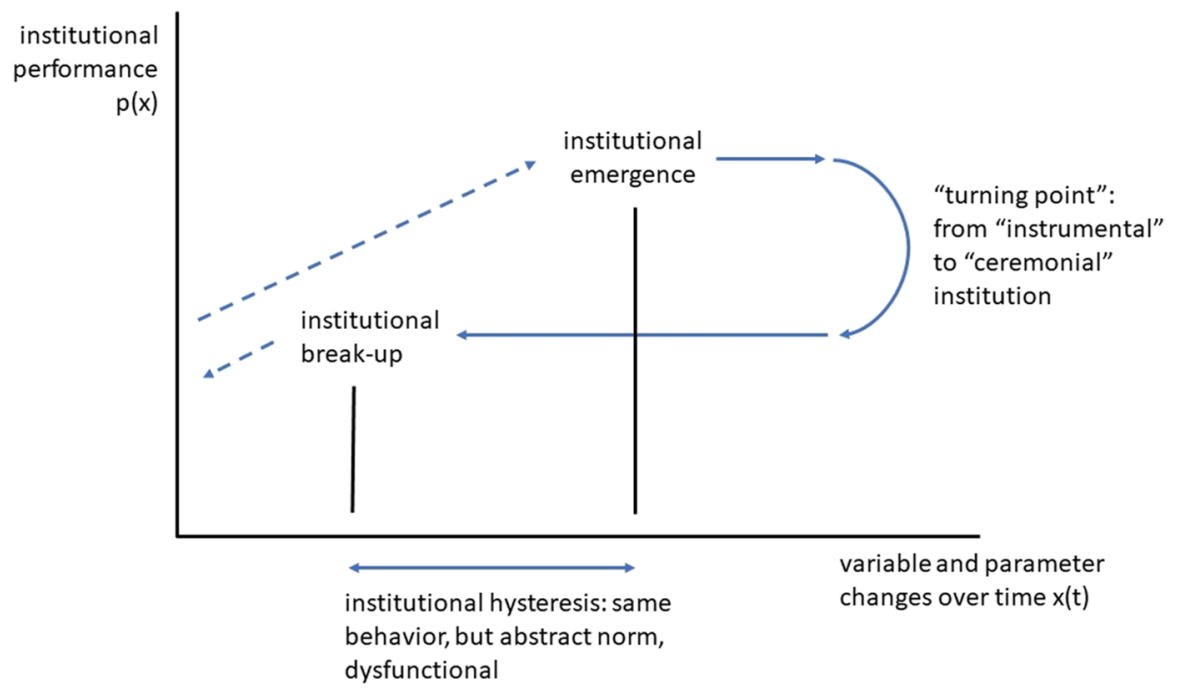

Fig. 1 Asymmetry of institutional emergence and collapse mapped on institutional instrumentality and performance

between emergence and collapse come into effect, under parameters moving gradually (linearly) back and forth? Sunk fixed costs suggest that institutions tend to exist longer than effective for high performance, and still exist under unfavorable circumstances, in which cooperation would not have emerged. For instance, the minimum critical mass of agents required to establish an institution may be larger than the one that keeps an old institution somehow working. For an illustration see Fig. 1.

The understanding of the endogeneity and asymmetry of emergence and break-up may benefit from insights developed in anthropology, where emphasis is put on the environment of developing socio-economies. The presence or absence of competing groups or societies influences the cohesiveness and fragility of a cooperative regime. This is the essence also of Turchin's (2003) argument on the emergence of large-scale cooperation through group-level competition and selection. In human evolution, selection favored prosocial, cooperative behavior within groups to an extent that is exceptional within the animal kingdom and cannot just be explained by genetic evolution. Rather, the emergence of human cultural capabilities facilitated processes of inter-group selection, helping particularly cooperative group cultures to gain competitive advantages over less cooperative ones. As a result, the human behavioral repertoire includes psychological dispositions for cooperation that were acquired in a co-evolutionary process of rising cultural capabilities and genetic adaptations required to live in cultural environments (e.g., Toth and Robinson 2007; Henrich 2016). The specific expression of those dispositions, however, depends on the cultural und institutional dynamics. The history of the last ca. 10,000 years provides very distinct solutions to large-scale coordination that may exhibit either cooperation or dominance (e.g., Traulsen and Nowak 2006; Wilson et al. 2013). This is consistent with the game-theoretic insight of multiple solutions for social dilemmas above the benchmark of complete defection, as given by the "folk theorem". 
Beyond such mechanisms of endogenous emergence, anthropology also provides evidence for asymmetry: the propensity to cooperate is asymmetric with respect to size. While small groups may spontaneously develop a cooperative culture, successful groups and populations that have grown into larger entities will have to rely on further provisions, particularly institutions that involve formal hierarchies. So, while institutions may emerge in small groups and persist in large ones through additional factors, institutional collapse may occur in large groups when those additional factors of stabilizing cooperation disappear (see Sects. 3 and 4).

Against this background, we state our two initial claims as hypotheses, which will be refined from the various perspectives in the following sections:

H1: Endogeneity: In the evolutionary process of emergence of institutionalized cooperation, there are mechanisms (in group psychological, cognitive, interaction- and network-related respects) that make institutional decline and collapse endogenously occur through institutional success.

H2: Asymmetry: Due to different habituated, group-based, and cultural-dispositional aspects of institutional emergence, institutional collapse may occur asymmetrically to (at different parameter constellations than) its emergence.

\section{The individual level: Psychological determinants of cooperation and its collapse}

Cooperation is conceptualized as a prosocial act toward another person or group, which comes at a cost for the acting individual. Because of their evolutionary history, humans are characterized by a fundamental motivational ambiguity towards their social counterparts (e.g., Boehm 1989; Eibl-Eibesfeldt 1997). This has been the foundation of the theory of individual behavior in fundamental works in economics since Adam Smith's Theory of Moral Sentiments (1759), and later, for instance, in evolutionary-institutional economics (Veblen 1899): on the one hand, there is a phylogenetical legacy of our primate ancestors that can make us behave agonistic and opportunistic (Eibl-Eibesfeldt 1997, 525-560). On the other hand, evolutionary trends in early hominids, enhanced by processes of cultural group selection of competing hominid bands (e.g., Richerson and Boyd 2005), lead to the genetic fixation of several genuine human prosocial capabilities, such as empathic behavior, sense of fairness, and spontaneous cooperation. Which part of human nature is actually displayed depends on the circumstances of social interaction, variance in personality, learning processes, and cultural influences (e.g., Tomasello 2009).

\subsection{Individual-level determinants for prosocial behavior and cooperation}

According to Tomasello (1999), the evolution of empathy was a major evolutionary step not only for the development of cultural learning abilities, but also for our natural inclination to act prosocially. In neuroscience, empathic reactions are studied by using neuro-imaging 
techniques in experiments that involve social interactions and provide hints for a physiological basis of cooperative behavior. According to Singer and Lamm (2009), an empathic reaction occurs when the observation of affective states in another person induces shared states in the observer. fMRI studies have investigated empathic responses in several emotional states including pain, anxiety, anger, pleasant affects, social exclusion, or embarrassment (see Bernhard and Singer 2012). These responses are taking place in brain regions (anterior insula, anterior and midcingulate cortex) that are usually activated if the person herself feels these emotions. Additionally, brain areas that are associated with social recognition are active in empathic situations. Empathy is distinct from emotional contagion or mimicry: while these do not require a separation from the source of the respective emotion (the self or the other), empathetic reactions involve affective sharing, self-awareness, and self-other distinction. Batson et al. (2007) argue that while observing a person suffering, the observer does not only represent the related pain within her own brain, but is motivated to relieve the suffering of the respective person, which translates into prosocial behavior. Hein et al. (2010) find that the induction of helping behavior is triggered by empathetic reactions. That spontaneous prosocial behavior in humans is present already with young infants has been shown in experimental studies (e.g., Bischof-Köhler 1991; Warneken and Tomasello 2009). This indicates that altruistic tendencies in humans reflect genetic predispositions.

Why do humans have genetic dispositions toward prosocial behaviors that are so vital for the emergence of cooperation? Ethology explains this via the rearrangement of functional behavioral modules during human evolution. Lorenz (1974) argues that prosocial emotions emerged originally by a redirection of initially aggressive drives (evoked by the social partner) toward a third party. The social partner serves as a "release mechanism" of aggressive psychic energy that is diverted to the environment, preferably to out-group persons. Recent ethologists argue that brood-caring instincts, which initially evolved in the context of childcare, are redirected toward the "extended family". The dyadic emotional dispositions between mother and child were extended first to a triadic relationship including the father and later on to the whole social group (Eibl-Eibesfeldt 1997, 525-560). Similarly, the "big-mistakehypothesis" in evolutionary psychology argues that in the course of increasing social abilities in humans, behaviors that evolved for kinship relations were extended to non-kin present in the social surrounding (Burnham and Johnson 2005).

\subsection{Individual-level determinants of opportunism and non-social behavior}

As indicated, prosocial behavior and its correlating brain structures are, in phylogenetic terms, relatively young. They are therefore competing with a more ancient emotional heritage of our primate ancestors, which presumably has been much more agonistic. While humans are able to extend their prosocial inclinations into an environment that is very different from the situation under which the social brain has evolved, there are also situations that trigger agonistic and opportunistic behavior or make it more likely. Indeed, several mechanisms at the group level evolved to keep these non-social tendencies under control (see Sect. 4). In the absence of such control, non-social behavior becomes much more likely. 
Knickmeyer et al. (2006) show that fetal testosterone levels are responsible for the degree of empathy a person can display in later life. Women seem to be on average more capable of empathic reactions than men are, because of lower testosterone production in sensitive phases of social brain development. Accordingly, men are more likely to show agonistic behavior. Baron-Cohen (2002) argues that above-average expression of testosterone in early-usually male-infancy may even lead to an absence of emphatic abilities. These persons then often have tremendous problems in their socialization and show usually reduced prosocial abilities. Bischof-Köhler (1991) argues that the ability to empathize can also induce malicious gloating if the personal relation (e.g., with an observed person in pain) is bad. Bernhard and Singer (2012) argue on the basis of fMRI studies that empathic reactions of people may be reduced if competing neuronal networks, e.g., the ones associated with emotions of revenge in the nucleus accumbens, are active.

In human ethology, it is argued that agonistic behavior is induced by the so called "fight and flight" system, suppressing prosocial behaviors (e.g., Eibl-Eibesfeldt 1997, 525-560). In situations of danger and stress, several neuro-active substances and hormones are released to push muscular activation in order to facilitate defense. This holds true for social stress as well (e.g., Jansen et al. 1995). One situation that may induce stress reactions is the presence of strangers. In children of a certain age, as well as in many native hunter-gatherer communities, out-groupers are not evoking spontaneous prosocial behavior, but are treated with caution or even fear. EiblEibesfeldt $(1997,242)$ argues that this is a universal trait that has to be modified by individual learning and cultural imprinting. Hein et al. (2010) find that the propensity to help in-group members via activation of empathic neuronal networks is increased compared to out-group members in adults.

A variety of in-group conflicts trigger agonistic behaviors, e.g., situations where a person is bullied or treated unfairly. In most cultures, people expect to be treated fair in social interaction and react with considerable willingness to punish defectors if these expectations are violated (e.g., Henrich et al. 2001; Fehr and Fischbacher 2003). This includes the costly punishment of someone who has treated a third person of the group in an unfair manner. Singer et al. (2006) find that empathic reactions are modulated by the perceived fairness of others. The strength of these reactions against a cheater receiving shocks is significantly reduced, which correlates with the willingness to punish unfair agents. The defense of fairness rules and the suppression of bullying is also connected to the human tendency to preserve an egalitarian social structure (e.g., Boehm 2001). ${ }^{3}$ Bullying behavior may be triggered by a personality that lacks empathic abilities, harsh environmental conditions and stress, or by opportunities to exploit in the absence of functioning social control mechanisms. Extreme forms of anti-social behavior, however, are often associated with reduced sizes of brain structures associated with emotions, such as the amygdala or the prefrontal cortex (Kiehl et al. 2001; Raine and Yang 2006). The question whether there exists a drive for aggressive behavior per se, producing spontaneous

\footnotetext{
3 The impact of hierarchy on spontaneous cooperation in organizations is studied in Witt/Schwesinger (2013).
} 
aggression, or if aggression is mainly instrumental to achieve other goals, is by no means settled in the literature (e.g., Eibl-Eibesfeldt 1997, 535).

From these findings it follows that individuals can be considered to show spontaneous cooperative and prosocial behaviors based on compassion and empathy and experienced as self-rewarding. People are more likely to show considerable degrees of prosocial behavior within their own social group, though, preferably against individuals they are emotionally attached to. However, this prosocial tendency is fragile: individual ontogeny and learning, stressful situations, crestfallen expectations concerning the behavior of others, as well as influences from the wider cultural background may trigger non-cooperative or even aggressive behaviors. We therefore expect that the ambivalent human nature facilitates cooperation in large-scale social systems if the institutional setting is robust enough to accommodate situations that would trigger opportunistic or anti-social responses. This is summarized by our first specific hypothesis:

H1.1: Humans have both a propensity for aggressive (selfish, non-cooperative) behavior and prosocial psychological predispositions. The latter provide a basis for large-scale cooperation, which is much more common toward perceived ingroup members. The willingness to behave cooperatively relies on expectations regarding mutual compliance with social institutions, such as equal treatment, and related norms, such as fairness and reciprocity. The violation of these institutions activates antagonistic cognitive modules, i.e., the propensity for aggressive behavior. This evokes costly punishment and exploitative behavior, finally causing cooperation to collapse.

In the next section, we will draw on these findings when analyzing the evolution of a firm's corporate culture as an example of a social institution.

\section{The group-level: Anthropological evidence on group-based cooperation and its collapse}

A central tenet of evolutionary theories is that behavior of organisms should maximize genetic fitness. As a corollary of this, natural selection should lead to cooperation among large numbers of individuals only if they are genetically closely related. With the exception of humans, where cooperation with non-relatives emerges spontaneously in small- and medium-sized groups, this result is consistent with available evidence (e.g. Mayr 1991). Large-scale cooperation is a kind of first choice only for human agents. For instance, in most organizations, employees cooperatively contribute much more to their organization's overall goal achievement than the minimum that could be extracted from them by supervisory enforcement of the (not fully specifiable) employment contract (e.g., Cordes et al. 2008; Carpenter et al. 2009). Yet, every organization faces the problem of the commons: benefits that are jointly gained and shared by all-non-contributors and contributors-and the resulting temptation of free-riding (e.g., Hardin 1968; Simon 1991). Why, then, is there 
anything besides free-riding? Why are humans capable of large-scale cooperation among non-relatives? And when does this large-scale cooperation break down?

\subsection{Cultural group selection and the emergence of group-bound cooperation}

The ancestors of modern humans became highly cultural in the Middle Pleistocene, perhaps 250,000 years ago (e.g., McBrearty and Brooks 2000). Humans are unique in the degree to which they depend upon culturally transmitted information to create complex adaptations (Norenzayan and Heine 2005). Boyd and Richerson (2002) proposed that the dispositions for cooperation and group-beneficial behaviors have evolved in a process of (1) gene-culture coevolution in combination with (2) cultural group selection (also Tomasello 2009; Henrich 2016): (1) the prevalent level of cooperation in a group as cultural arrangements exert some selection on innate human social dispositions. Culturally evolved social environments favor an innate psychology that is suited to these environments, such as a psychology aiming at social rewards and avoiding social sanctions (e.g., Rilling et al. 2002; Reuter et al. 2011). Moreover, repeated gene-culture coevolutionary cycles established more and more complex social institutions of cooperation in groups based on these evolved cognitive features. Over many generations this coevolutionary dynamic generated a social psychology that facilitates cooperation and other prosocial behaviors. These cognitive dispositions are the coevolved products of genes responding to the social and moral environments created by cultural institutions. This selective mechanism involves quite different behaviors from those favored by selection on genes alone. (2) By producing multiple behavioral equilibria among groups that comprise group-beneficial equilibria, cultural evolution endogenously facilitated a mechanism of selection among groups that favored prosociality (Henrich 2004; Soltis et al. 1995): cultural group selection selected from alternative behavioral equilibria in favor of the ones most successful in competing with other groups. In this context, groups with higher prosocial norms including cooperation had an advantage over groups with more competitive internal cultures.

Many aspects of group life root in this evolutionary past of humans (Robson and Kaplan 2003; Henrich, 2016). Their evolved cognitive dispositions-including prosocial behaviors-adapted for living in groups. The more complex societies of the sort we live in today only began to evolve about 5000 years ago, too little time for much evolution of the genetic aspects of our social psychology. Hence, contemporary complex societies are based upon the cultural evolution of institutions using our social predispositions that resulted from the processes described. Anthropology argues that human nature is fundamentally tribal (see Richerson et al. 2006). Consequently, the group is critical to an understanding of human behavior and the workings of society's institutions. Individual dispositions to cooperate can be sufficient, in the first instance, to initiate and sustain cooperation in small-scaled groups using informal face-to-face interactions. Moreover, these social predispositions include a tendency to identify with larger, symbolically marked groups and their institutions and norms (e.g., McElreath et al. 2003). Large, complex global societies, therefore, still depend upon the dispositions that help stabilize cooperation in small groups as their building blocks (e.g., Richerson and Boyd 2005). 
Anthropologist Coon (1946) argued that small groups, in which people know one another personally and meet and communicate habitually, form the fundamental units of human organization. The only successful way to organize human agents in larger, more complex societies is through combination of small groups as faceto-face organizations. ${ }^{4}$ Essential organizational processes are grounded in personto-person relationships of in-group members that are not part of formal control systems. Also, studies on village-scale commons management suggest that small, band-based systems can be maintained by informal agreements, but that larger systems may require formal rules and mechanisms as well, such as formal monitoring and sanctioning (Ostrom 2000) — and need to be built from basic modules as a layered multiplex network structure (e.g., Simon 1962; Wilson et al. 2013; Sect. 6). Thus, the band-sized group represents the limits of cooperation organized by purely informal means. Larger groups that produce significant benefits have clearly articulated (formal) institutions for monitoring and enforcement.

\subsection{Conditions of deteriorating cooperation and collapse: Group size and growth}

As has been shown in Sect. 3, cooperation in groups is fragile, highly contextual, and depends on mutual compliance with social institutions (also Henrich et al. 2006; Herrmann et al. 2008). In this context, a critical factor is group size and its growth. A common finding in lab experiments and field data is that, as groups grow, performance declines: people who belong to larger groups are, other things equal, less satisfied with group membership, participate less often in collective activities, show more misbehavior, free riding, and contribute less often to group activities (Levine and Moreland 1990; Mukhopadhaya 2003; Forsyth 2006). Coordination problems through increasing uncertainty, decreasing trust, and motivation losses in larger, more anonymous groups with higher (perceived) complexity, opacity, and volatility (in meeting interaction partners) impair performance and prevent reaping productive potentials.

Marlowe (2005) reviewed group sizes among hunter-gatherers who most closely resembled our Pleistocene ancestors. Local residential groups (bands) averaged 48 (median 30) people, i.e., hunter-gatherer bands tended to equilibrate at group sizes around less than 50 individuals. These groups were nested within ethno-linguistic groups (tribes), whose sizes averaged 1750 members. The author found no indication of local-group sizes depending on other factors than cognition, such as, e.g., natural resources. Rather, the upper limit on group size appears to be determined by the frequency of bickering, reflecting an increase in free riding. This interpretation is further strengthened by the results of Dunbar (1993, 2008), who highlights the existence of cognitive constraints on our ability to maintain social, personalized relationships at a given level of emotional intensity (see Sawaguchi and Kudo 1990; Zhou et al. 2005). This manifests in the structure of the human brain: average species' group size correlates with relative neocortex size. The derived expected critical sizes of human groups correspond well with the observations of Marlowe.

\footnotetext{
${ }^{4}$ See also Simon's (1962) theory of the modular architecture of complex systems.
} 
These constraints are still relevant today. Anecdotal evidence is given by the existence of tailored packages of management consultancy that aim at firms reaching a critical size at 50-60 employees. ${ }^{5}$ Firms below this threshold can be operated in more freewheeling, informal ways and face-to-face contacts by an influential business leader who maintains a cooperative corporate culture (Cordes et al. 2008). Above that number, a change toward more conventional management practices or transition to smaller subdivisions is needed (Witt 1998). This implies that to sustain cooperation in growing groups (particularly more than 50 people) requires (1) more explicit and formal institutions, control, and sanctioning or (2) sophisticated structures of subdivisions. For instance, when asked in an interview how to maintain Google's corporate culture while the organization is growing, the firm's founders argued for the existence of a "natural size for human organizations" and that creating (sub-)groups of this size "...can retain a lot of that culture" (Lashinsky, 2008).

These findings suggest that the collapse of cooperation occurs endogenously when group sizes increase without these counter measures being put in place (also Elsner and Schwardt 2014). It becomes more difficult in larger groups to realize institutionalized cooperation and related social and economic performance. Greater group size easily gives rise to basic changes in members' behavior: more frequent appearance of opportunistic behavior in growing groups allows for the rapid actualization of nonsocial predispositions and the spreading of selfish behavior (see Sect. 3). Members who were willing to contribute to the benefit of the group may suddenly change their behavior when the group exceeds a critical size (see Gladwell 2000; Card et al. 2008).

These insights from anthropology have implications for collapse and emergence of cooperation:

H1.2: Endogeneity: In the course of cooperation-driven growth of groups, these reach a cognitive critical size beyond which the level of cooperation among their members may rapidly decline and collapse. Group size and the related economic entity are then likely to shrink again.

Moreover, once opportunistic or free-riding behavior becomes an established behavioral alternative to cooperation, a return to large-scale cooperation at the grouplevel is unlikely to happen. This leads to the following specific asymmetry hypothesis:

H2.1: Asymmetry: The tipping-point of size-induced collapse of institutions of cooperation within groups is asymmetric to its point of emergence. When groups decline, the former level of cooperation will probably not be reached again. Levels of cooperation and organizational unit sizes as well as socio-economic performance will therefore remain for longer periods below the threshold (of parameter settings) of earlier cooperation emergence.

An illustrating case in point is the evolution of a corporation's culture (see Fig. 2). In small firms, a cooperative culture can be established by informal means of management, e.g., cognitive leadership (Witt 1998), that tap humans' pro-social disposition to cooperate (Cordes et al. 2008).

\footnotetext{
${ }_{5}^{5}$ See, e.g., http://www.ipso-facto.biz.
} 


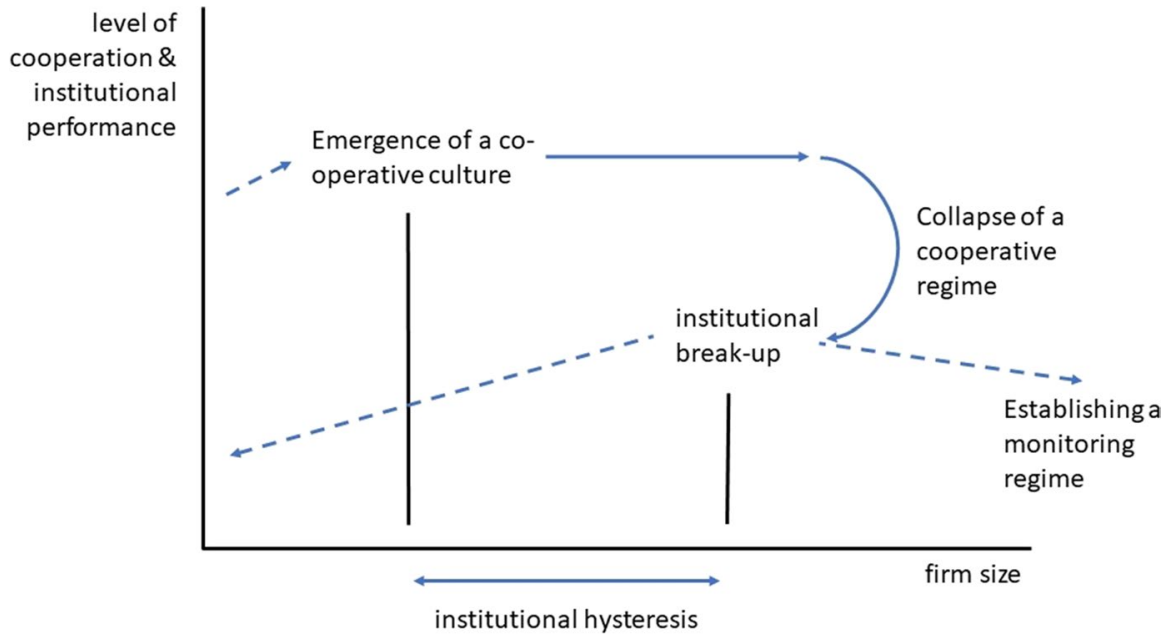

Fig. 2 The asymmetry of emergence and collapse of cooperation in firms

A cooperative regime significantly contributes to firm success, e.g., in terms of growth in employment, especially in nascent, turbulent business environments. However, when reaching a critical size, the organization faces a growth crisis. Absent organizational and managerial reforms (see above), cooperation collapses as a result of endogenous firm growth. Moreover, once group members are lost to the selfish, opportunistic camp, they are unlikely to return to cooperation: even if a shrinking organization reaches the size at which cooperation originally emerged, this kind of culture is most probably not reestablished - an instance of institutional hysteresis. This process also gives rise to a path-dependent asymmetry of emergence and collapse of an (informal) institution in historical time. Firm decline due to increasing opportunism and finally exit may represent institutional break-up. Alternatively, a firm may implement a monitoring regime that keeps in check opportunism within the organization. It facilitates further growth in employment, however, at lower levels of institutional performance due to the hysteretic drop in cooperation.

\section{Emergence and collapse of cooperation in networks}

Cooperation emerges more easily in closely connected small groups due to a relatively high probability to meet again. In practice, however, cooperative groups can be quite large. This is because interactions are structured by social networks: typically, an agent in a population does not interact with every other agent, but only with a certain subset. Therefore, Dunbar's (1993) cognitive critical group-size remains valid in larger and more complex population networks and is applicable to their smaller sub-populations. In this way, the structure of social networks represents a critical factor when it comes to the evolution of cooperation: the properties of a population's interaction structure, particularly 
its degree distribution, clustering, average path-length, and the interrelations among those three, have important implications for the emergence and collapse of cooperation.

\subsection{Network structures and the emergence of cooperation}

The theoretical significance of networks has been stressed in a number of modelbased studies. Most find that the dynamics of emergence and collapse of cooperation are different on non-trivial (or 'complex') networks as compared to the dynamics on complete networks (Zenou 2012). In these models, agents learn and adapt to the environment by copying strategies of more successful agents in their vicinity (Fu et al. 2008; Fehl et al. 2011; Santos et al. 2012). A number of network properties that facilitate the emergence of cooperation have been identified in this context, the most relevant of which are heterogeneous degree distributions and high degree correlations.

High degree correlation is a property of a network according to which highdegree nodes (hubs) tend to have high-degree neighbors. This facilitates the spreading of cooperation between high-degree nodes (e.g., Rong and Wu 2009). With lower degree-correlation, a network would have a substantial number of isolated hub-structures, in which defective behavior may more easily persist. Moreover, heterogeneous degree distributions facilitate cooperation by generating a negative feedback for defecting hubs, and a positive feedback for cooperative hubs (e.g., Santos et al. 2012; Pacheco et al. 2009). Defecting hubs, i.e., defectors with many connections, cause their less well-connected neighbors to copy their defective strategy although well-connected neighbors will be less easily compelled to become defectors. The spread of defection to the neighborhood reduces the hub's success and forces it to copy the cooperative strategy from other hubs it remains connected to. Heterogeneous degree distributions are common given that most social networks are scale-free (see Barabási and Bonabeau 2003).

Endogenous modifications of networks may also facilitate the emergence of cooperation. Choosing, avoiding, or cutting links to particular interaction partners facilitates attaining an initial critical minimum mass $\mathrm{k}$ (or ratio $\mathrm{k} / \mathrm{n}$ ) of cooperating agents or might allow for ostracism as a cheap way to punish defecting agents. This enables cooperators to generate superior payoffs overall and, thereby, take over the population and entailing social or spatial clustering among cooperators (e.g., Mantzaris et al. 2013). However, the mere fact that a network is dynamic does not inherently facilitate the emergence of cooperation on it. Network dynamics that are neutral or detrimental to the emergence of cooperation are conceivable as well.

\subsection{Network structures and the collapse of cooperation}

A change of the network structure toward a less favorable parameter setting, e.g., through the reduction of a high degree correlation, can hinder the emergence of 
cooperation. But can it also lead to the collapse of a cooperative population? As to the effect of network structure, there is evidence that points to a clear asymmetry between emergence and collapse of cooperation. For the case of degree correlation, Rong and Wu (2009) found that a high degree correlation favors the emergence of cooperation, yet a low degree correlation stabilizes the current strategy configuration. Moreover, if a network is clustered, a cooperative group may be able to maintain itself as a cluster within a less and less favorable broader environment for some time. But if the environmental conditions deteriorate further, the group collapses and this collapse will be sudden and catastrophic. The group will quickly reintegrate with the rest of the population, once the mechanisms that protected the group vanish. The phenomenon has become known recently as Seneca effect, i.e., mechanisms entailing slow emergence but rapid collapse (Bardi 2017) and can conveniently be modeled within a catastrophe theory model (Heinrich 2018). Empirical social networks are highly clustered and subject to dynamic modifications that favor clustering. The described asymmetry is, therefore, expected to occur frequently and strongly. The deterioration of favorable conditions for cooperation may be caused by a variety of exogenous changes. One example, for which the effect has been demonstrated, is changing resource availability (Sugiarto et al. 2015). In any case, changing the network structure that has facilitated the emergence of cooperation does not immediately comes with its breakdown, indicating that they are one important reason for the asymmetry of emergence and collapse of cooperation.

\subsection{The relevance of networks structures}

The interaction structure of a population as represented by a network has important implications. Hence, within the overall systemist approach pursued in this paper, the description of any system should include a description of its internal network structure. Not surprisingly, network structure has important implications for the asymmetry between emergence and collapse of cooperation, as summarized in the following hypotheses. They refer to a system with a network structure in which changing environmental conditions should lead to an emergence of cooperation at parameter set $\mathrm{A}$, but to the collapse of cooperation, if change is reversed, at point B (see Fig. 3).

H1.3: Endogeneity (partner selection) facilitates the emergence of cooperation by shifting both $\mathrm{A}$ and $\mathrm{B}$ in favor of the emergence and persistence of cooperation.

H1.4: Endogeneity (partner selection) facilitates the emergence of cooperation relatively more than the collapse of cooperation.

H2.2: Asymmetry: For networks with homogeneous degree distribution, points A (emergence) and B (collapse) are stable and identical. For networks with heterogeneous degree distribution, $\mathrm{A}$ and $\mathrm{B}$ will be stable and significantly different from one another. 
a) Networks with homogeneous degree distributions

Emergence of cooperation

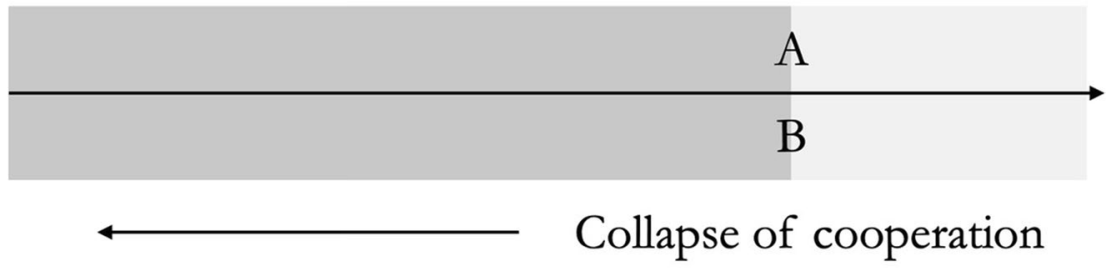

b) Networks with heterogeneous degree distributions

Emergence of cooperation

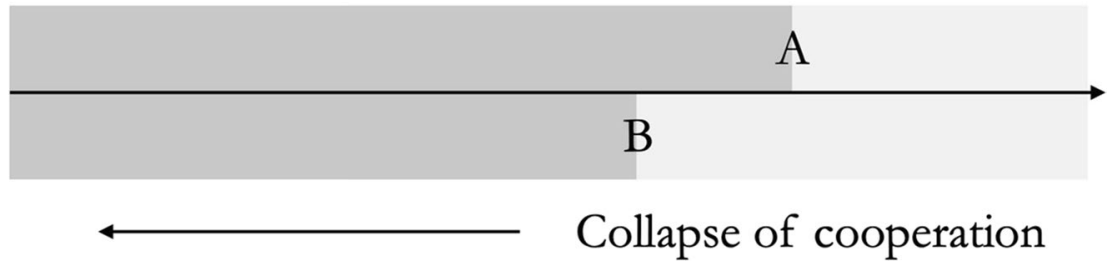

Fig. 3 The asymmetry of emergence and collapse of cooperation in networks with homogeneous and heterogeneous degree distributions according to H2.2. Once cooperation has emerged, making the population parameters less favorable to cooperation leads to a collapse that is different to the point of emergence for networks with heterogeneous degree distributions

\section{An evolutionary-institutional perspective on endogeneity and asymmetry}

\subsection{Emergence of cooperation}

The puzzle of cooperation gained attention in economics in the context of the private provision of collective goods in the 1960s (Runciman and Sen 1965; Olson and Zeckhauser 1967). Interest in cooperation further developed via behavioral experiments and game theorists who incorporated psychological, biological, and anthropological evidence into their models (Axelrod 1984; Bowles and Gintis 2013). The emergence of cooperation is usually based on iterated prisoners' dilemmas in an evolution-of-cooperation approach. Interpreting this result within the framework of evolutionary-institutional theory, cooperation is not feasible based on myopic hyperrationality. It rests on interactively learned and habitually applied social institutions followed by boundedly rational agents and on learned expectations to meet an interaction partner again or, more generally, to meet a cooperator in a population. With learned and habituated cooperation in some interaction arena, spill-overs into other 
arenas, where agents interact in other positions and with other partners, become feasible (Gintis 2004; Bednar et al. 2015). Institutionalization, habituation, and normatization anchor cooperative behavior beyond myopic calculus and choice. Institutionalized cooperative behavior then becomes part of the broader social environment (Bowles and Gintis 2013; McCain 2014). Agents may cooperate even toward strangers in one-shot interactions.

In basic EGT, emergence and collapse of cooperation would formally appear symmetric and simply be reversals. Collapse would occur at the same set of values of, e.g., the discount factor or population shares, where emergence did before. But the institutional embeddedness of behavior allows to consider asymmetries between emergence and collapse.

\subsection{Factors of institutional emergence bear endogeneity of collapse}

Emerged institutionalized cooperation bears economic success through its superiority over common defection, for both the individual and the relevant group. Nevertheless, the individualist incentive to defect and exploit remains dominant in the short run. The successful, cooperating population may grow in income and in numbers, be it through more progeny or higher attraction to outsiders and increasing immigration. This expansion may deteriorate the very cognitive conditions of cooperation. In a growing population, newcomers may not learn incumbent cooperative culture sufficiently fast. The cooperators' share in the population may fall below a minimum critical mass. Perceived uncertainty, over-complexity, and opacity may increase again. This implies a loss of recognized interdependence, of knowledge on problem-solving, of the perceived connection between action and reaction, or of a sufficiently long-run perspective (Glaeser et al. 2002; Solari and Gambarotto 2014). With returning myopia, the incentive to realize the short-run payoff becomes the dominant motivation again. Diamond (2005, 427-431) calls such backsliding into "rational bad behavior" the "most frequent" reason of collapse.

Another reason for the endogeneity of collapse may be the uneven distribution of the gains of cooperation under differential hierarchical status, power, or network centrality. The ceremonial motive of more differential status and power may strengthen the mechanism to unevenly distribute the gains in favor of the more powerful. The empirical evidence for the relevance of inequality as a cause of socio-economic collapse is large (e.g., Turchin 2003; Furman 2017). Distributional aspirations for higher relative socioeconomic position may then dominate over absolute achievement in a society. The more distributional aspirations dominate, the less socially mobile the society and the worse overall performance often will become (e.g., Veblen 1899 on positional struggle in predatory societies; Torgler et al. 2006; Wilson et al. 2013; Nishi et al. 2015).

Sticking to the old institution, then, is attractive for those agents who find themselves in the favored position. But, as C.E. Ayres (1962) pointed out, the dominance of ceremonial institutional behavior tends to lead even agents who do not benefit to continue following the established institutional habits. The habituated behavior provides institutionalized identity and belongingness to the lower ranks of the group (Brewer and Kramer 1986; Elsner 2012). Furthermore, habits save cognitive effort 
and match it with cognitive capacity and keep monitoring, punishment, and other transaction costs relatively low. The formally same habituated behavior will in those cases be exercised with different consequences, including a shift to a non-cooperative character, to exploitative relations, reduced socio-economic performance, and eventually economic decline, crisis, and collapse.

\subsection{Factors of emergence also bear asymmetry}

Cognitive limits and habituation also apply to the case of asymmetry. This relates to the fact that coordinating people onto a newly emerging institution is time-consuming and costly. This may apply even beyond the point where conditions (parameters) have reversed to the same state where the institution initially emerged (e.g., Elsner 2012; Heinrich and Schwardt 2013). The inferior, now inappropriate, old institution will then be upheld beyond the point of instrumentality. Under both variants of institutional inappropriateness, (1) dominance of the ceremonial dimension causing an increasing unevenness of distribution and a change in incentive structures and (2) ineffectiveness as to institutional economies of scale, an asymmetry of institutional collapse vis-à-vis its emergence is expected. Be it that it is upheld beyond the point of appropriate change or beyond the point of earlier emergence should the conditional parametrical configuration simply reverse.

Due to such institutional hysteresis, there may be a period of stagnancy, while the final collapse is assumed to be relatively sudden. Further, once a culture of noncooperation spreads, it will be aggravated by increased monitoring costs and more costly punishment (Horiuchi 2015). Too fierce a punishment culture may also prevent innovation and further the degeneration of the institutional setting. High degrees of punishment are negatively correlated with cooperation, problem-solving, and socioeconomic thriving (e.g., Povey 2014). Other related costs then increase as well, such as costs of building reputation, signaling, contracting, controlling, insuring, etc. (Fu et al. 2008). Real-world examples of collapse result in increasing short-termism (e.g., Aspara et al. 2014) and winner-takes-all cultures, both entailing decreasing system resilience **(e.g., Acemoglu et al. 2015).

This evolutionary-institutional argument can be summarized by the following hypotheses:

H1.3: Endogeneity: Institutions of cooperation endogenously collapse if (1) their success makes the relevant group or population grow, which deteriorates the cognitive conditions required for cooperation through higher volatility, myopia, and opacity, perceived over-complexity, and higher monitoring and punishment costs. (2) Increasingly unequal distribution of the gains of cooperation in power- and status-asymmetries makes the same formal institutional behavior ceremonial. Cooperation eventually breaks up and defection and distributive and positional struggle take effect.

H2.5: Asymmetry: Institutional collapse occurs asymmetrically, i.e., at less favorable levels of parameter sets than emergence when (1) redistributive processes and transformed incentives not only make the winners stick to institutionalized 
behavior, but inferiors as well, who yield stability, identity, and belongingness, matching cognitive limits as to the pace of institutional change. (2) Institutionalized behavior is used beyond the constellation of its emergence when parameters reverse in order to save cognitive effort by habituation and normatization and to exploit decreasing costs of collective decision-making of the old institution.

\section{Conclusions}

In this paper, we developed an integrative multiparadigmatic and interdisciplinary perspective on the collapse of cooperative institutions and their related socio-economies. We focused on mechanisms of the (1) endogeneity of institutional collapse and (2) the asymmetry between conditions of emergence and collapse. At this point, we integrate these insights from different paradigms and disciplines within the greater theoretical framework of 'systemism' (Bunge 2004; Gräbner and Kapeller 2017). It provides a unified ontological vantage point according to which each element of reality is considered either as a system and/or a part of one. Each system itself is described via the CESM model, which provides a unified vocabulary for the integration of theories. For an arbitrary system $\sigma$, a model $\mu(\sigma)$ comprises a list $\langle C(\sigma), E(\sigma), S(\sigma), M(\sigma)\rangle$, in which $C(\sigma)$ lists its constituent parts, $E(\sigma)$ the environmental variables, $S(\sigma)$ the networks structure, and $M(\sigma)$ the mechanisms. Our analysis of the emergence and collapse of cooperation looks at systems at different levels of analysis, which themselves are parts of systems at higher levels of aggregation. The theories described in Sects. 3 and 4 are mainly concerned with the micro and meso level, where the individuals, which are the main subjects of investigation of Sect. 3, form the parts of the systems considered in Sect. 4. At the same time, we described 'bridges' between the levels, such as multilevel selection mechanisms, which link the different layers of analysis. These take the form of bottom-up or top-down mechanisms. Section 5 focused on the role of network structure $S(\sigma)$, which is relevant on various levels of aggregation. Section 6, then, was mainly concerned with the mechanisms of institutional change, which serve as bottom-up and top-down bridging mechanisms between higher and lower ontological levels of analysis. This way, the different contributions are integrated in one consistent framework and yield a number of conclusions.

First, in the biological and anthropological perspective of gene-culture coevolution, our psychological endowment as a heritage of human social evolution provides us with some disposition to behave prosocially and cooperatively (Sect. 3). This is linked to an expectation that a partner will comply to rules of fairness, reciprocity, and equal treatment. If this expectation is violated, humans tend to switch to a more agonistic behavior. If interactions violate our expectation or norm set, our propensity to cooperate is strongly reduced. We endogenously (i.e., within the dispositional spectrum available), but often asymmetrically (e.g., under more non-cooperation than prevalent when the institution emerged), switch back to a phylogenetically more ancient propensity of aggressive (non-cooperative) behavior. Particularly, cooperation is connected to a perception of "in-group" vs. "out-group" and may collapse through (1) growth of the relevant group or (2) intensifying interactions with 
out-groups, which both cannot be sufficiently cognitively absorbed by the in-group members.

Second, this is in line with findings from anthropology (Sect. 4). Evolution and multi-level selection at individual, in-group, and between-group levels was a central driver of large-scale cooperation. Under strong between-group rivalry, cooperative institutions are favored by cultural group selection. However, the attenuation of external rivalry may relax the need to maintain these institutions entailing institutional decline and collapse in some groups (the relevant population).

Third, network structures can influence emergence and collapse of cooperation (Sect. 5). Among the characteristics that have been identified as favorable to cooperation are heterogeneous degree distributions, high degree correlations, and some endogenous link formation and removal dynamics. Further, conditions of emergence and collapse of cooperation on non-trivial networks are not typically symmetric. Instead, cooperative structures may, once in existence, be able to support themselves even under conditions unfavorable to their initial emergence. This capacity is limited: reaching this limit leads to a catastrophic collapse, not a gradual one mirroring emergence.

Fourth, also an evolutionary-institutional analysis (Sect. 6) reveals constellations where we find endogenous and asymmetric collapse. The very success of cooperation may lead to prosperity and growth of the group, which, in turn, may increase perceived turbulence and endogenously undermine the cognitive and expectational foundations of cooperation. Further, cooperation gains may be unevenly distributed, which also may endogenously undermine established cooperation. There is ample empirical, experimental, and computational knowledge that inequality may cause institutional and socio-economic decline and collapse (e.g., Elsner 2017). In all, increasing turbulence, rising inequality and ceremonialization, entailing myopia, higher monitoring and punishment costs, may lead to a collapse of cooperation and socio-economic prosperity.

In terms of asymmetry, the very sunk fixed costs of institutional emergence, particularly habituation, psychological generalization, and normatization, imply decreasing average costs of collective decision-making and thus a tendency to apply an institution beyond the point where it became inappropriate and a new one should have emerged. And even if the set of parametric conditions linearly reverses, the values originally prevalent at emergence may already be undershot when collapse eventually occurs. The overlong maintenance of the old institution makes it ever less problem-solving and lead the economy into decline over a period of time, while the institutional collapse may occur suddenly when institutions experience cumulative crisis.

Considering processes after collapse, it appears unlikely that a group will easily recover to re-emergence and previous levels of cooperativeness. This is known in the theoretical, historical, and empirical literature, e.g., for societies suffering from exclusive institutions (Acemoglu and Robinson 2012). Collapse of cooperation proved to be non-reversible for long periods, another asymmetry with the previous emergence and the foregoing collapse. We conclude that not only the process of emergence but also of collapse of prosociality is fundamentally endogenous to complex adaptive systems and their evolution. It is asymmetric in different disciplinary and paradigmatic perspectives. A largely consistent system of hypotheses could be derived, which can be used in further research for more detailed complex modeling 
and (empirically anchored) computational research. The overall framework of systemism provides the adequate meta-theoretical language to align this research within a common framework of analysis. It is not only for theoretical and formal, but also for urgent contemporary practical reasons that economics should increasingly turn to problems of institutional and systemic collapse.

Funding Open Access funding enabled and organized by Projekt DEAL.

\section{Appendix}

\section{See Table 1.}

Table 1 Definitions of key terms

\begin{tabular}{|c|c|}
\hline formal social institution & $\begin{array}{l}\text { Socially approved and practiced behavioral rule for solving collective- } \\
\text { decision problems; learned in an evolutionary interaction process; norm } \\
\text { endowed with a willingness to sanction deviators and to inform everyone } \\
\text { that with ubiquitous deviation all will be worse-off than with general abid- } \\
\text { ance }\end{array}$ \\
\hline Formal social institution & $\begin{array}{l}\text { Collectively decided, codified and prescribed behavioral rule endowed with a } \\
\text { formal sanction through state / policy punishment }\end{array}$ \\
\hline Social rule & $\begin{array}{l}\text { Socially approved and practiced behavioral regularity for solving coordina- } \\
\text { tion problems; learned in an evolutionary interaction process; has become a } \\
\text { norm informing everyone that with unilateral deviation the deviator will be } \\
\text { worse-off than with abidance }\end{array}$ \\
\hline Culture/cultural trait & $\begin{array}{l}\text { Culture is considered to be all of the information that individuals acquire } \\
\text { from others by a variety of social learning processes including teaching } \\
\text { and imitation; a cultural trait is defined as an idea, norm, belief, attitude, } \\
\text { habit, or value that is acquired by social learning and that influences an } \\
\text { individual's behavior; system of informal social institutions }\end{array}$ \\
\hline Organization & $\begin{array}{l}\text { System of formal and informal institutions among which super-/subordina- } \\
\text { tion is critical }\end{array}$ \\
\hline Cooperation & $\begin{array}{l}\text { Prosocial act toward a group that entails a cost for the acting individual; an } \\
\text { altruistic behavior }\end{array}$ \\
\hline
\end{tabular}

\section{Declarations}

Conflict of interest The authors declare that they have no conflicts of interests.

Open Access This article is licensed under a Creative Commons Attribution 4.0 International License, which permits use, sharing, adaptation, distribution and reproduction in any medium or format, as long as you give appropriate credit to the original author(s) and the source, provide a link to the Creative Commons licence, and indicate if changes were made. The images or other third party material in this article are included in the article's Creative Commons licence, unless indicated otherwise in a credit line to the material. If material is not included in the article's Creative Commons licence and your intended use is not permitted by statutory regulation or exceeds the permitted use, you will need to obtain permission directly from the copyright holder. To view a copy of this licence, visit http://creativecommons.org/licen ses/by/4.0/. 


\section{References}

Acemoglu D, Robinson JA (2012) Why nations fail. The origins of power, prosperity and poverty. Profile Books, London

Acemoglu D, Ozdaglar A, Tahbaz-Salehi A (2015) Networks shocks, and systemic risk. In: Oxford handbook on the economics of networks. Columbia Business School research paper, pp. 15-18.

Aspara J, Pajunen K, Tikkanen H, Tainio R (2014) Explaining corporate short-termism: self-reinforcing processes and biases among investors, the media and corporate managers. Soc Econ Rev 12(4):667-693

Axelrod R (1984) The evolution of cooperation. Basic Books, New York (repr.2006)

Ayres CE (1962) The theory of economic progress. Foreword to the 1962 edition, UMKC internet edition, http://cas.umkc.edu/econ/Institutional/Readings/Ayres/tep/TEP.html

Barabási A, Bonabeau E (2003) Scale-free networks. Sci Am 288(5):60-69

Bardi U (2017) The Seneca effect. Why growth is slow but collapse is rapid. Springer, Heidelberg

Baron-Cohen S (2002) The extreme male brain theory of autism. Trends Cogn Sci 6(6):248-254

Batson CD, Eklund JH, Chermok VL, Hoyt JL, Ortiz BG (2007) An additional antecedent of empathic concern: valuing the welfare of the person in need. J Pers Soc Psychol 93(1):65-74

Bednar J, Jones-Rooy A, Page SE (2015) Choosing a future based on the past: Institutions, behavior, and path-dependence. Eur J Polit Econ 40:312-332

Bernhardt BC, Singer T (2012) The neural basis of empathy. Annu Rev Neurosci 35:1-23

Bischof-Köhler D (1991) The development of empathy in infants. In: Lamb ME, Keller H (eds) Infant development: Perspectives from German speaking countries. Lawrence Erlbaum Associates, Hillsdale, NJ, pp 245-273

Block F (2000) Disorderly co-ordination: the limited capacities of states and markets. In: Burlamaqui L et al (eds) Institutions and the role of the state. Elgar, Northampton, pp 53-71

Boehm C (1989) Ambivalence and compromise in human nature. Am Anthropol 91:921-939

Boehm C (2001) Hierarchy in the forest: the evolution of egalitarian behavior. Harvard University Press, Cambridge

Bowles S, Gintis H (2013) A cooperative species. Princeton University Press, Princeton

Boyd R, Richerson PJ (1985) Culture and the evolutionary process. University of Chicago Press, Chicago

Boyd R, Richerson PJ (2002) Group beneficial norms can spread rapidly in a structured population. J Theor Biol 215(3):287-296

Brewer MB, Kramer RM (1986) Choice behavior in social dilemmas: effects of social identity, group size, and decision framing. J Pers Soc Psychol 50(3):543-549

Brooks N (2012) Beyond collapse: climate change and causality during the Middle Holocene Climatic Transition, 6400-5000 years before present. Danish J Geogr 112(2):93-104

Bunge M (2004) How does it work? The search for explanatory mechanisms. Philos Soc Sci $34(2): 182-210$

Burnham TC, Johnson DD (2005) The biological and evolutionary logic of human cooperation. Analyse Kritik 27(2):113-135

Card D, Mas A, Rothstein J (2008) Tipping and the dynamics of segregation. Quart J Econ 123(1):177-218

Carpenter J, Bowles S, Gintis H, Hwang SH (2009) Strong reciprocity and team production: theory and evidence. J Econ Behav Organ 71(2):221-232

Coon CS (1946) The universality of natural groupings in human societies. J Educ Sociol 20(3):163-168

Cordes C, Richerson PJ, McElreath R, Strimling P (2008) A naturalistic approach to the theory of the firm: the role of cooperation and cultural evolution. J Econ Behav Organ 68(1):125-139

Diamond J (2005) Collapse. How societies choose to fail or succeed. Viking-Penguin, New York

Dunbar RIM (1993) Co-evolution of neocortex size, group size and language in humans. Behav Brain Sci $16(4): 681-735$

Dunbar RIM (2008) Cognitive constraints on the structure and dynamics of social networks. Group Dyn Theory Res Pract 12(1):7-16

Eibl-Eibesfeldt I (1997) Die Biologie des menschlichen Verhaltens. Seehamer Verlag, Weyarn

Elsner W (2012) The theory of institutional change revisited. the institutional dichotomy, its dynamic, and its policy implications in a more formal analysis. J Econ Issues 41(1):1-43

Elsner W (2017) Complexity economics as heterodoxy: Theory and policy. J Econ Issues 51(4):939-978 
Elsner W, Schwardt H (2014) Trust and arena size: expectations, institutions, and general trust, and critical population and group sizes. J Inst Econ 10:107-134

Elster J (1983) Explaining technical change. A case study in the philosophy of science. Cambridge University Press, Cambridge

Epstein J, Axtell R (1996) Growing artificial societies. Social science from the bottom up. MIT Press, Cambridge

Fehl K, Van der Post DJ, Semmann J (2011) Co-evolution of behaviour and social network structure promotes human cooperation. Ecol Lett 14(6):546-551

Fehr E, Fischbacher U (2003) The nature of human altruism. Nature 425(6960):785-791

Forsyth DR (2006) Group dynamics. Thomson Wadsworth, Belmont

Fu F, Hauert C, Nowak MA, Wang L (2008) Reputation-based partner choice promotes cooperation in social networks. Phys Rev E 78(2):026117

Furman J (2017) Productivity is slowing and inequality is growing. Productivity, inequality, and economic rents. http://evonomics.com/productivity-is-slowing-inequality-is-growing-heres-whatscausing-it/. Accessed 24 Apr 2017

Gintis H (2004) The genetic side of gene-culture coevolution: internalization of norms and prosocial emotions. J Econ Behav Organ 53(1):57-67

Gladwell M (2000) The tipping point. Little, Brown and Company, Boston

Glaeser E, Laibson D, Sacerdote B (2002) An economic approach to social capital. Econ J 112(483):F437-F458

Gräbner C, Kapeller J (2017) The micro-macro link in heterodox economics. In: Jo T-H, Chester L, D'Ippolliti C (eds) The handbook of heterodox economics. Routledge, London, pp 145-159

Hardin G (1968) The tragedy of the commons. Science 162(3859):1243-1248

Hein G, Silani G, Preuschoff K, Batson CD, Singer T (2010) Neural responses to ingroup and outgroup members' suffering predict individual differences in costly helping. Neuron 68(1):149-160

Heinrich T (2018) A discontinuity model of technological change: catastrophe theory and network structure. Comput Econ 51(3):407-425

Heinrich T, Schwardt H (2013) Institutional inertia and institutional change in an expanding normal-form game. Games 4:398-425

Henrich J (2004) Cultural group selection, coevolutionary processes and large-scale cooperation. J Econ Behav Organ 53(1):3-35

Henrich J (2016) The secret of our success. Princeton University Press, Princeton, NJ

Henrich J, Boyd R, Bowles S, Camerer C, Fehr E, Gintis H, McElreath R (2001) In search of homo economicus: behavioral experiments in 15 small-scale societies. Am Econ Rev 91(2):73-78

Henrich J, McElreath R, Barr A, Ensminger J, Barrett C, Bolyanatz A, Cardenas JC, Gurven M, Gwako E, Henrich N, Lesorogol C, Marlowe F, Tracer D, Ziker J (2006) Costly punishment across human societies. Science 312:1767-1770

Herrmann B, Thöni C, Gächter S (2008) Antisocial punishment across societies. Science 319:1362-1367

Hodgson GM (2000) From micro to macro: the concept of emergence and the role of institutions. In: Burlamaqui L et al (eds) Institutions and the role of the state. Elgar, Northampton, pp 103-126

Horiuchi S (2015) Emergence and collapse of the norm of resource sharing around locally abundant resources. J Artif Soc Soc Simul 18(4):7

Jansen AS, Van Nguyen X, Karpitskiy V, Mettenleiter TC, Loewy A, A. D. (1995) Central command neurons of the sympathetic nervous system: basis of the fight-or-flight response. Science 270(5236):644-646

Janssen MA, Kohler TA, Scheffer M (2003) Sunk-cost effects and vulnerability to collapse in ancient societies. Curr Anthropol 44(5):772-728

Kiehl KA, Smith AM, Hare RD, Mendrek A, Forster BB, Brink J, Liddle PF (2001) Limbic abnormalities in affective processing by criminal psychopaths as revealed by functional magnetic resonance imaging. Biol Psychiat 50(9):677-684

Knickmeyer R, Baron-Cohen S, Raggatt P, Taylor K, Hackett G (2006) Fetal testosterone and empathy. Horm Behav 49(3):282-292

Lashinsky A (2008) Back2Back champs-larry page, sergey brin, and eric schmidt on keeping the culture alive - and how they all promised to stay at google for 20 years. Fortune 157(2):70

Levine JM, Moreland RL (1990) Progress in small group research. Annu Rev Psychol 41:585-634

Lorenz K (1974) On aggression. Houghton Mifflin Harcourt, Boston, MA

Mantzaris AV, Basset DS, Wymbs NF, Estrada E et al (2013) Dynamic network centrality summarizes learning in the human brain. Journal of Complex Networks 1:83-92 
Marlowe FW (2005) Hunter-Gatherers and human evolution. Evol Anthropol 14:54-67

Mayr E (1991) One long argument: charles darwin and the genesis of modern evolutionary theory. Harvard University Press, Cambridge

McBrearty S, Brooks AS (2000) The Revolution that wasn't: a new interpretation of the origin of modern human behavior. J Hum Evol 39(5):453-563

McCain RA (2014) Reframing economics. Economic action as imperfect cooperation. Elgar, Northampton

McElreath R, Boyd R, Richerson PJ (2003) Shared norms and the evolution of ethnic markers. Curr Anthropol 44(1):122-129

Meija J (2018) The network formation origin of tribal societies. Documento CEDE, no. 33.

Mukhopadhaya K (2003) Jury size and the free rider problem. J Law Econ Organ 19(1):24-44

Nishi A, Shirado H, Rand D, Christakis N (2015) Inequality and visibility of wealth in experimental social networks. Nature 526(7573):426

Norenzayan A, Heine SJ (2005) Psychological universals: what are they and how can we know? Psychol Bull 131(5):763-784

Olson M Jr, Zeckhauser R (1967) Collective goods, comparative advantage, and alliance efficiency. In: Issues in defense economics, NBER, pp. 25-63

Ostrom E (2000) Collective action and the evolution of social norms. J Econ Perspect 14(3):137-158

Pacheco JM, Pinheiro FL, Santos FC (2009) Population structure induces a symmetry breaking favoring the emergence of cooperation. PLoS Comput Biol 5(12):e1000596

Povey R (2014) Punishment and the potency of group selection. J Evol Econ 24(4):799-816

Raine A, Yang Y (2006) Neural foundations to moral reasoning and antisocial behavior. Social Cognit Affect Neurosci 1(3):203-213

Rasmus J (2016) Systemic fragility in the global economy. Clarity Press, Atlanta, GA

Reuter M, Frenzel C, Walter NT, Markett S, Montag C (2011) Investigating the genetic basis of altruism: the role of the COMT Val158Met polymorphism. Soc Cognit Affect Neurosci 6(5):662-668

Richerson PJ, Boyd R (2005) Not by genes alone: how culture transformed human evolution. The University of Chicago Press, Chicago

Richerson PJ, Collins D, Genet RM (2006) Why managers need an evolutionary theory of organizations. Strateg Organ 4(2):201-211

Richman BD (2016) An autopsy of cooperation: diamond dealers and the limits of trust-based exchange. http://scholarship.law.duke.edu/faculty_scholarship/3594/, Accessed 25 Apr.

Rilling JK, Gutman DA, Zeh TR, Pagnoni G, Berns GS, Kilts CD (2002) A neural basis for social cooperation. Neuron 35:395-405

Robson AJ, Kaplan HS (2003) The evolution of human life expectancy and intelligence in Hunter-Gatherer economies. Am Econ Rev 93(1):150-169

Rong Z, Wu ZX (2009) Effect of the degree correlation in public goods game on scale-free networks. Europhys Lett 87(3):30001

Runciman WG, Sen AK (1965) Games, justice and the general will. Mind 74(296):554-562

Santos FC, Pinheiro FL, Lenaerts T, Pacheco JM (2012) The role of diversity in the evolution of cooperation. J Theor Biol 299:88-96

Sawaguchi T, Kudo H (1990) Neocortical development and social structure in primates. Primates 31(2):283-289

Setterfield M (1996) Hysteresis and Uncertainty. Complementary or competing visions of evolving economic systems? In: Pressman S (ed) Interactions in political economy. Routledge, London, pp $133-148$

Simon HA (1962) The architecture of complexity. Proc Am Philos Soc 106:467-482

Simon HA (1991) Organizations and markets. Journal of Economic Perspectives 5(2):25-44

Singer T, Lamm C (2009) The social neuroscience of empathy. Ann NY Acad Sci 1156(1):81-96

Singer T, Seymour B, O’Doherty JP, Stephan KE, Dolan RJ, Frith CD (2006) Empathic neural responses are modulated by the perceived fairness of others. Nature 439(7075):466-469

Smith A (1759) The Theory of Moral Sentiments. Millar, Kincaid, and Bell. Edinburgh

Solari S, Gambarotto F (2014) Territorial rooting as an element of well-being. Rev Soc Econ 72(4):504-522

Soltis J, Boyd R, Richerson PJ (1995) Can group-functional behaviors evolve by cultural group selection? An empirical test. Curr Anthropol 36(3):473-494

Sugiarto HS, Chung NN, Lai CH, Chew LY (2015) Socioecological regime shifts in the setting of complex social interactions. Phys Rev E 91:062804 
Tainter JA (2003) The collapse of complex societies. Cambridge University Press, New York

Tainter JA (2006) Archaeology of overshoot and collapse. Annu Rev Anthropol 35:59-74

Tomasello M (1999) The human adaptation for culture. Annu Rev Anthropol 28:509-529

Tomasello M (2009) Why we cooperate. MIT Press, Boston

Torgler B, Schmidt SL, Frey BS (2006) Relative income position and performance: an empirical panel analysis. In: FEEM working paper, vol. 39, Yale Center for International and Area Studies, New Haven, CT, USA

Toth AL, Robinson GE (2007) Evo-devo and the evolution of social behavior. Trends Genet 23(7):334-341

Traulsen A, Nowak MA (2006) Evolution of cooperation by multilevel selection. Proc Natl Acad Sci 103(29):10952-10955

Turchin P (2003) Historical dynamics: why states rise and fall. Princeton University Press, Princeton, NJ

Turchin P, Currie T, Turner E, Gavrilets S (2013) War, space, and the evolution of Old World complex societies. Proc Natl Acad Sci 110(41):16384-16389

Veblen TB (1899) The theory of the leisure class: an economic study of institutions. Macmillan, New York

Warneken F, Tomasello M (2009) The roots of human altruism. Br J Psychol 100(3):455-471

Weiss H, Bradley RS (2001) What drives societal collapse? Science 291:609-610

Wilson DS, Ostrom E, Cox ME (2013) Generalizing the core design principles for the efficacy of groups. J Econ Behav Organ 90S:S21-S32

Witt U (1998) Imagination and Leadership — the neglected dimension of an evolutionary theory of the firm. J Econ Behav Organ 35(2):161-177

Witt U, Schwesinger G (2013) Phylogenetic footprints in organizational behavior. J Econ Behav Organ 90:S33-S44

Zenou Y (2012) Networks in Economics. In: CEPR discussion papers, no. 9021, appeared in: international encyclopedia of the social and behavioral sciences

Zhou W-X, Sornette D, Hill RA, Dunbar RIM (2005) Discrete hierarchical organization of social group sizes. Proc R Soc B 272:439-444

Publisher's note Springer Nature remains neutral with regard to jurisdictional claims in published maps and institutional affiliations.

\section{Authors and Affiliations}

${\text { Christian } \text { Cordes }^{1} \text { (D) } \text { Wolfram Elsner }}^{1}$. Claudius Graebner ${ }^{2,3}$. Torsten Heinrich $^{4}$ • Joshua Henkel ${ }^{1}$. Henning Schwardt ${ }^{5}$. Georg Schwesinger ${ }^{1}$. Tong-Yaa Su ${ }^{1}$

1 Faculty of Business Studies and Economics, University of Bremen, P.O. Box, 28359, Bremen, Germany

2 Institute for the Comprehensive Analysis of the Economy (ICAE), Johannes Kepler University, Linz, Austria

3 Institute for Socio-Economics, University of Duisburg, Essen, Germany

4 Institute for New Economic Thinking (INET), Oxford Martin School, University of Oxford, Oxford, UK

5 Department of Economics, University of Denver, Denver, CO, USA 